\title{
EFFECTS OF LIP REVISION SURGERY ON LONG-TERM OROSENSORY FUNCTION IN PATIENTS WITH CLEFT LIP/ PALATE
}

\author{
Greg Essick, D.D.S., Ph.D., \\ Professor, Department of Prosthodontics, University of North Carolina at Chapel Hill. \\ Ceib Phillips, M.P.H., Ph.D., \\ Professor, Department of Orthodontics, University of North Carolina at Chapel Hill. \\ Yunro Chung, M.S., and \\ Ph.D. Candidate, Department of Biostatistics, University of North Carolina at Chapel Hill.

\section{Carroll-Ann Trotman, B.D.S., M.A., M.S.} \\ Professor, Department of Orthodontics, The University of Maryland School of Dentistry, \\ Baltimore, Maryland, and Adjunct Professor, Department of Orthodontics, The University of North \\ Carolina School of Dentistry, Chapel Hill, North Carolina.
}

\begin{abstract}
Objective-To determine whether secondary lip revision surgery impacts sensitivity of the upper lip.

Design-A three-group, parallel, prospective, nonrandomized clinical trial.

Setting-University of North Carolina School of Dentistry.

Patients, Participants-Three groups: (1) patients with repaired cleft lip/palate who were scheduled for lip revision (revision; $\mathrm{n}=20$ ); (2) patients with repaired cleft lip/palate who did not receive a lip revision (non-revision; $\mathrm{N}=13$ ); and (3) non-cleft control subjects (non-cleft; $\mathrm{N}=22$ ).

Interventions-Lip revision surgery.

Main Outcome Measures-Measures of (1) two-point perception threshold, (2) warmth detection threshold (warm), and (3) cool detection threshold (cool) were obtained from two sites on the upper lip vermilion. The revision participants were tested approximately 1 week before surgery and then approximately 3 and 12 months after surgery. The non-revision and non-cleft participants were tested at similar times.
\end{abstract}

Results-There were no significant differences among the three groups at baseline for two-point, warm, or cool. The main effects of group, age, sex, and time were not statistically significant for the two-point or warm $(P>.05)$. The mean differences between the 3- and 12-month follow-up visits and baseline for two-point and warm were small for all three groups. For cool, group was statistically significant $(P=.04)$, the difference in the non-revision group between follow-up and baseline was $31 \%$ to $34 \%$ higher than in the non-cleft group $(P=.01)$.

Conclusions-Although at postsurgery revision participants exhibited threshold values comparable to presurgical values, the sensory differences observed among subgroups of participants with cleft lip are complex.

(C) Copyright 2012 American Cleft Palate-Craniofacial Association

Address correspondence to: Dr. Carroll-Ann Trotman, 650 West Baltimore Street, Dean's Suite \#6408, Baltimore, MD, 21201. 


\section{Keywords}

cleft lip; lip revision; lip sensitivity

Most neurosensory studies published to date suggest that patients with cleft lip and palate (cleft lip/palate) exhibit normal or near normal orofacial sensory function. For example, tactile sensitivity (pressure detection thresholds) and acuity (two-point discrimination) on the upper lip, mid-face, and hard and soft palates have been found to be comparable, on average, for groups of patients and control subjects (Posnick et al., 1994; Uchiyama et al., 1998; Akal et al., 2000; Rousseau et al., 2011). Moreover, threshold values for two-point perception, warmth detection, and cool detection on the facial skin and vermilion of the upper lip have been found similar for patients and control subjects (Essick et al., 2007; Rousseau et al., 2011). However, these studies compared the sensitivity either between noncleft control subjects and patients with cleft lip/palate years after the initial lip surgery or between previously repaired and non-cleft sides of the face in patients with cleft lip/palate. The purpose of the analyses reported in this study was to determine if additional (secondary) revision surgery impacts sensitivity of the upper lip within the first year after the surgery. Three key questions were addressed: (1) Do neurosensory assessments (two-point perception and thermal thresholds) on upper vermilion sites differ for participants with a cleft lip who receive secondary revision surgery versus those who do not? (2) For patients receiving revision surgery, do neurosensory assessments differ at 3 and 12 months post surgery? (3) For patients receiving revision surgery, do neurosensory assessments differ from those of non-cleft controls at either 3 or 12 months post surgery?

\section{Methods}

The data reported below were obtained from participants in a longitudinal study of the functional outcomes of lip-revision surgery (C.T., principal investigator). The study design was a parallel, nonrandomized clinical trial (Trotman et al., 2007) with three subject groups: (1) patients with repaired cleft lip/palate who were scheduled for lip revision (revision); (2) patients with repaired cleft lip/palate who did not receive a lip revision either because they declined revision surgery or were not referred for the surgery (non-revision); and (3) noncleft control" subjects (non-cleft). The lip revision surgery was performed at the recommendation of the surgeon after consultation with the patient and parents. The surgical technique has been described previously (Trotman et al., 2007).

Study participants were screened and recruited in clinics at the University of North Carolina School of Dentistry. The inclusion criteria were: (1) parent and patient willingness to participate; (2) an ability to comprehend verbal instructions; and (3) for the patients, a previously repaired complete unilateral or bilateral cleft lip with or without a cleft palate. The exclusion criteria were: (1) previous orthognathic or facial soft-tissue surgery; (2) a medical history of diabetes, collagen vascular disease, and/or systemic neurologic impairment; (3) mental or hearing impairment such that comprehension or ability to perform tests was hampered; and (4) for the patients, a lip revision surgery within the past 2 years. The Institutional Review Board approved the study, and written informed consent and assent were obtained. For the purpose of this analysis, only participants 12.5 years of age or older at enrollment were included. Participants younger than 12.5 years were excluded because of difficulties they experienced in successfully completing the testing protocol.

The revision participants were tested approximately 1 week before the lip revision surgery and then again at approximately 3 and 12 months after the surgery. These postsurgery testing times are important to track changes related to the regenerative process, and these 
times are supported by a large body of literature (Karas et al., 1990; Van Boven and Johnson, 1994; Fridrich et al., 1995). Subjects in the non-revision and non-cleft groups were group-frequency matched to the revision group on the basis of age and gender and tested at similar intervals. At each appointment, estimates for the (1) two-point perception threshold, (2) warmth detection threshold (warm), and (3) cool detection threshold (cool) were obtained from two sites on the upper lip vermilion. Each site was located halfway between the midline and commissure of the lips at rest, with no consistent relationship to visible scars when present. The sequence of site testing was randomized for each threshold procedure. In a few instances, the testing was terminated early due to the participant's lack of attention or inability to understand instructions, resulting in missing data for a site or the entire procedure. Each participant kept his/her eyes closed during estimation of the thresholds.

\section{Two-point Perception Threshold}

The two-point perception threshold (2PPT) was estimated at each test site using a tracking procedure based on maximum likelihood estimation (Chen et al., 1995; Feldman et al., 1997). The stimuli were provided by a disk-shaped Disk-Criminator (Neuro Regen LLC, Lutherville, MD), supporting multiple sets of two small metal prongs at 16 different separations from 2 to $25 \mathrm{~mm}$ (Chen et al., 1995). On each trial, the experimenter pressed the two prongs perpendicularly into the skin for 2 to 3 seconds without producing discomfort or lateral movement. The prongs were oriented horizontally along the vermilion site under study. After the stimulus was removed, the participant responded either "yes" ("two touches" were felt) or "no" (only "one touch" was felt). The threshold-tracking algorithm specified the separation to be used based on the participant's previous responses. At each visit, 15 trials were administered, and the threshold-tracking algorithm then calculated the 2PPT on the basis of the separation predicted to result in the perception of two points 50\% of the time. The 2PPT at each visit was $\log 10$ transformed, and the difference between each follow-up visit and baseline was calculated. A positive difference indicated that a greater separation was required for perceiving the separation as two points at the follow-up visit, consistent with impaired performance.

\section{Warmth and Cool Detection Thresholds}

A modification of the Marstock protocol was used to estimate the detection thresholds for warmth and cool at each site (Fruhstorfer et al., 1976; Essick et al., 2004). The warmth (thermal) stimuli were delivered by a TSA-II NeuroSensory Analyzer (Medoc Advanced Medical Systems, Durham, NC). A special intraoral transducer probe, $6 \mathrm{~mm}$ in diameter, was used to apply thermal stimuli to the discrete sites on the vermilion. A single layer of cling film was stretched tightly across the end of the probe to maintain clinical asepsis. The film was replaced between subjects. Prior to measuring the thermal thresholds, the temperature of the vermilion was estimated using a remote sensing infrared thermometer held above the test sites (SenseLab Tempett; Somedic Sales AB, Hörby, Sweden). This temperature defined the vermilion temperature. If needed, cotton rolls were placed in the labial vestibule to improve access to the vermilion.

To test each site, the transducer probe was set to the vermilion temperature and applied perpendicularly to the skin with consistent and comfortable pressure (the probe indented the vermilion skin 1 to $2 \mathrm{~mm}$ ). After about 7 seconds, warming pulses were produced at $0.3^{\circ} \mathrm{C} /$ sec. Pulses were terminated by the participant's response to signify that the transducer began to feel warmer. The temperature returned to the vermilion temperature (VT), and the next stimulus was initiated at a randomly chosen time between 4 and 6 seconds. Four stimuli were delivered at each test site. If the participant's responses to the first two stimuli appeared inappropriate to the experimenter (e.g., the participant responded before the warming pulse was delivered), the testing was restarted at that site. The warmth detection 
threshold at each visit was calculated as the difference between the mean of the four response temperatures and the vermilion temperature (mean response - VT). Positive thresholds indicated that warmth was detected at temperatures above the vermilion temperature. The warmth detection threshold at each visit was $\log 10$ transformed, and the difference between the $\log 10$ value at each follow-up visit and baseline was then calculated. A positive difference at follow-up indicated an increase in the temperature before warmth was detected at the follow-up visit, consistent with impaired performance.

The cool detection threshold was estimated in a similar manner. The stimulus temperature decreased at $0.3^{\circ} \mathrm{C} / \mathrm{sec}$ until the participant responded to signify that the transducer began to feel cool. The cool detection threshold was calculated at each visit as the difference between the vermilion temperature and the mean of the four response temperatures (VT - mean response). Thus, positive thresholds indicated that cooling was detected at temperatures below the vermilion temperature. The cool detection threshold at each visit was $\log 10$ transformed, and the difference between the $\log 10$ value at each follow-up visit and baseline was then calculated. A positive difference between visits indicated that the temperature perceived as cool was lower at the follow-up visit, consistent with impaired performance.

For the revision and non-revision groups, the affected (cleft) side threshold value was analyzed for subjects with a unilateral cleft, while the average of the right and left sides was used for subjects with a bilateral cleft. For the non-cleft group, the average of the right and left side threshold values was analyzed.

\section{Statistical Analysis}

An analysis of variance was performed to assess whether the average age at enrollment or the average thresholds at baseline differed among the three groups. To test for differences in the proportion of female patients among the three groups and bilateral cleft lip and cleft palate representation between the non-revision and revision groups, the Fisher exact test was used.

For each threshold, a linear mixed model with pairwise interactions for group by age, group by sex, and group by time using a compound symmetry covariance structure was used to compare the three groups with respect to the difference between 3 months and baseline and 12 months and baseline. The model was defined as:

$$
\begin{aligned}
& Y_{i j k}=\beta_{0}+b_{0 i}+\beta_{1} g_{1 k}+\beta_{2} g_{2 k}+\beta_{3} a g e_{i k}+\beta_{4} f_{i k}+\beta_{5} t i m e_{j}+\beta_{6} g_{1 k} a g e_{i k}+\beta_{7} g_{2 k} a g e_{i k}+ \\
& \beta_{8} g_{1 k} f_{i k}+\beta_{9} g_{2 k} f_{i k}+\beta_{10} g_{1 k} t^{t i m e} e_{j}+\beta_{11} g_{2 k} t i m e_{j}+e_{i j k}
\end{aligned}
$$

where $Y_{i j k}$ is the $\log 10$ difference between the outcome and baseline at jth time from ith participant in kth group $\left(i=1, \ldots . n_{k}, j=1\right.$ (3 month-baseline), $j=2$ (12 month-baseline), $k=$ 1 (control), $k=2$ (non-revision), $k=3$ (revision)); $\left(g_{1 k}, g_{2 k}\right)=(1,0)$ for control, $\left(g_{1 k}, g_{2 k}\right)=$ $(0,1)$ for non-revision, $\left(g_{1 k}, g_{2 k}\right)=(0,0)$ for revision; time $j=1$ for 3 months, time $=0$ for 12 months; $f_{i k}=1$ for female, $f_{i k}=0$ for male; $e_{i j k} \sim N\left(0, \sigma^{2}\right)$ and $b_{0 i} \sim N\left(0, \mathrm{~T}^{2}\right.$ are assumed to be independent. A backward selection approach was used to assess the interaction terms. All parameters were estimated using restricted maximum likelihood with Kenward-Roger adjusted degrees of freedom as implemented by SAS Proc Mixed (Version 9.2, SAS Institute Inc., Cary, NC; Kenward and Roger, 1997).

\section{Results}

Participants were recruited from May 2001 to September 2005, and follow-up was extended to November 2006. The three groups of participants did not differ significantly with respect to age $(P=.65)$ or sex $(P=.43)$ (Table 1$)$. The percent of subjects with bilateral cleft did not 
differ significantly between the non-revision and revision groups $(P=1.0)$, nor did the percent with a cleft palate in the two groups differ significantly $(P=.66)$.

The descriptive statistics for the differences in the $\log 10$ values for the two-point perception, the warm, and the cool thresholds by group are given in Table 2, as well as the $\log 10$ threshold values at baseline. The three groups did not differ significantly, on average, at baseline for two-point perception $(P=.65)$, warm $(P=.44)$, or cool $(P=.77)$ thresholds.

The pairwise interactions were not statistically significant for any of the thresholds and were removed from the model $(P>.07)$. None of the main effects (group, age, sex, and time) were statistically significant (Table 3 ) for the two-point perception (Fig. 1) or warmth detection thresholds (Fig. 2). The mean differences between each of the follow-up visits (3 and 12 months) and baseline for two-point perception were quite small for all three groups. The estimated antilog values based on the main effects model ranged from 0.893 to 1.059 , indicating that the mean thresholds at 3 and 12 months differed from the mean baseline values by $<11 \%$ (Fig. 1). Similarly, for each of the three groups, the mean warmth detection thresholds at follow-up differed from baseline by less than 19\% (Fig. 2).

For the cool detection thresholds, group was statistically significant $(P=.04)$, while age, sex, and time were not (Table 3). Pairwise comparisons between groups indicated that the non-revision group cool threshold difference between follow-up and baseline, on average, was significantly different from the non-cleft group $(P=.01)$, while the non-revision and revision groups $(P=.16)$ and the revision and non-cleft groups $(P=.19)$ were not significantly different. The difference between each follow-up visit and baseline was greatest in the non-revision group, approximating $31 \%$ to $34 \%$ higher than at baseline (Fig. $3)$.

\section{Discussion}

This study demonstrated that over the 12-month observation period, group mean variation in two-point perception, warmth detection, and cool detection was modest, and differences among the three groups of subjects were observed only for the cool detection threshold. Because the greatest difference from baseline ( 0 month) was observed for the non-revision group and the performance of all three groups was similar at 3 and 12 months, it is unlikely that the revision surgery had any impact on cool detection. The reason that the performance of the non-revision group rather than the revision group was affected is not clear. The mean cool detection threshold for the control group at baseline was relatively high, and decreased at 3 and 6 months. In contrast, the mean threshold for the non-revision group at baseline was relatively low, and increased at 3 and 6 months. Thus, the statistically significant difference between these two groups of participants may represent regression toward the means. Accepting the lack of a better explanation for the difference between these two groups at 3 and 12 months, of more importance is the observation that those participants who received revision surgery exhibited threshold values at 3 and 12 months post surgery comparable to those observed presurgically. Moreover, the three groups of participants did not differ significantly at baseline in the two-point perception, warmth detection, or cool detection. As such, the results of the present study are consistent with the literature that suggests that threshold measures of sensory function are normal on the lips of patients with cleft lip (and/ or palate; Posnick et al., 1994;Uchiyama et al., 1998;Akal et al., 2000; Essick et al., 2007; Rousseau et al., 2011).Also, group mean differences between patients with cleft lip and non-cleft controls at standardized test sites, as used in the present and most previous studies, are rarely observed even in patients during the first year after secondary revision surgery. 
In contrast to the studies cited above, a few investigators have reported an abnormality in orofacial sensory function in patients with cleft lip (and/or palate). Specifically, decreased sensitivity to vibration has been observed on the hard palate at the premolar teeth, suggesting injury to the greater palatine nerve (Uchiyama et al., 1998); however, other investigators have reported increased sensitivity to vibration on the nasolabial and labial skin (Posnick et al., 1994). In a recently published analysis, which included a subset of the participants of the present study, we found that although patients with cleft lip did not differ from non-cleft controls in threshold measures of warmth detection, cool detection, and twopoint perception, subgroups of participants with cleft lip exhibited differences in the thresholds (Essick et al., 2007). These differences could not be explained by a simple loss of innervation in and around the healed surgical sites. For example, lower thresholds for warmth detection were observed on the upper vermilion in participants with bilateral cleft lip compared to participants with unilateral cleft lip; however, for participants with unilateral cleft lip, the warmth threshold was not lower on the cleft side than on the non-cleft side. Lower thresholds for warmth detection were also observed on the vermilion in participants with cleft lip who reported altered sensation on the mid-face region compared to those who did not. The same participants exhibited higher thresholds for cool detection, compared to participants who did not report altered sensation on the mid-face.

In the same study, patients whose altered sensations were more consistent with hyposensitivity exhibited higher thresholds than patients whose altered sensations were more consistent with hypersensitivity; however, given that the thresholds did not differ between the cleft and non-cleft sides of the upper lip, the higher thresholds could not be explained solely by greater tissue and nerve injury. All considered, it was concluded that the sensory differences observed between subgroups of participants with cleft lip are complex and are not explained by any simple model purporting higher thresholds at less innervated sites.

A strength of the present study is that sensory function was measured before and longitudinally after lip revision surgery. These measurements were compared to those from patients with repaired cleft lip/palate who did not receive a lip revision surgery as well as from non-cleft control subjects. Previous studies typically have compared the sensitivity either of non-cleft control subjects to patients with cleft lip/palate years after the initial lip surgery or between previously repaired and non-cleft sides of the face in patients with cleft lip/palate.

A potential weakness of the present study is that sensitivity was measured only at fixed sites on the upper vermilion, and that the relationship between the location of these sites and the presence of cleft scars was not consistent across subjects. In a previous study, we found that altered sensation extended minimally from the scar in about half of the patients evaluated, but extended substantially away from the visible scar in the other patients (Essick et al., 2005). Thus, it is unknown whether the results of the present study would have been different if sensitivity had been measured directly over areas of visible scaring. Finally, the possibility of variable attention or of a full understanding of when to respond is always a concern with neurosensory testing of children. However, in a previous study we found that the threshold values obtained from non-cleft control children at sites on the vermilion of the lip were similar to the values we published previously from adult subjects, attesting to the validity of the data from younger subjects (Essick et al., 2007).

\section{Acknowledgments}

This research was supported by the University of Maryland School of Dentistry and NIDCR Grant DE013814. The content is solely the responsibility of the authors and does not necessarily represent the official views of the National Center for Research Resources or the National Institutes of Health. 


\section{References}

Akal UK, Sayan NB, Aydogan S, Yaman Z. Evaluation of the neurosensory deficiencies of oral and maxillofacial region following surgery. Int J Oral Maxillofac Surg. 2000; 29:331-336. [PubMed: 11071233]

Chen C, Essick GK, Kelly DG, Young MG, Nestor JM, Masse B. Gender-, side and site-dependent variations in perioral spatial resolution. Arch Oral Biol. 1995; 40:539-548. [PubMed: 7677600]

Essick GK, Dorion C, Rumley S, Rogers L, Young M, Trotman CA. Report of altered sensation in patients with cleft lip. Cleft Palate Craniofac J. 2005; 42:178-184. [PubMed: 15748109]

Essick GK, Guest S, Martinez E, Chen C, McGlone F. Site-dependent and subject-related variations in perioral thermal sensitivity. Somatosens Mot Res. 2004; 21:159-175. [PubMed: 15763901]

Essick GK, Phillips C, Trotman CA. Functional outcomes of cleft lip surgery. Part IV: Between- and within-participant variables affecting lip vermillion sensory thresholds. Cleft Palate Craniofac J. 2007; 44:624-634. [PubMed: 18177194]

Feldman JA, Essick GK, Zuniga JR, Phillips C. Inter-examiner reliability of three subjective clinical neurosensory tests. Int J Adult Orthod Orthognath Surg. 1997; 12:273-285.

Fridrich KL, Holton TJ, Pansegrau KJ, Buckley MJ. Neurosensory recovery following the mandibular bilateral sagittal split osteotomy. J Oral Maxillofac Surg. 1995; 53:1300-1306. [PubMed: 7562195]

Fruhstorfer H, Lindblom U, Schmidt WG. Method for quantitative estimation of thermal thresholds in patients. J Neurol Neurosurg Psychiatry. 1976; 39:1071-1075. [PubMed: 188989]

Karas ND, Boyd SB, Sinn DP. Recovery of neurosensory function following orthognathic surgery. J Oral Maxillofac Surg. 1990; 48:124-134. [PubMed: 2299455]

Kenward MG, Roger JH. Small sample inference for fixed effects from restricted maximum likelihood. Biometrics. 1997; 53:983-999. [PubMed: 9333350]

Posnick JC, Al-Qattan MM, Pron GE, Grossman JAI. Facial sensibility in adolescents born with cleft lip after undergoing repair in infancy. Plast Reconstr Surg. 1994; 93:682-685. [PubMed: 8134426]

Rousseau P, Zimmerman S, Schupp W, Schmelzeisen R, Otten JE. Sensibility changes of the infraorbital nerve after primary surgery on patients with unilateral cleft lip according to J. Delaire [in French]. Ann Chir Plast Esthet. 2011

Trotman CA, Phillips C, Essick GK, Faraway JJ, Barlow SM, Losken HW, van Aalst J, Rogers L. Functional outcomes of cleft lip surgery. Part I: Study design and surgeon ratings of lip disability and need for lip revision. Cleft Palate Craniofac J. 2007; 44:598-606. [PubMed: 18177192]

Uchiyama T, Nakano Y, Koeda H. Measurement of palatal surface sensation by neuro-sensory tests in the post-operative cleft palate patients. Bull Tokyo Dent Coll. 1998; 39:243-249. [PubMed: 10218005]

Van Boven RW, Johnson KO. A psychophysical study of the mechanisms of sensory recovery following nerve injury in humans. Brain. 1994; 117:149-167. [PubMed: 8149208] 


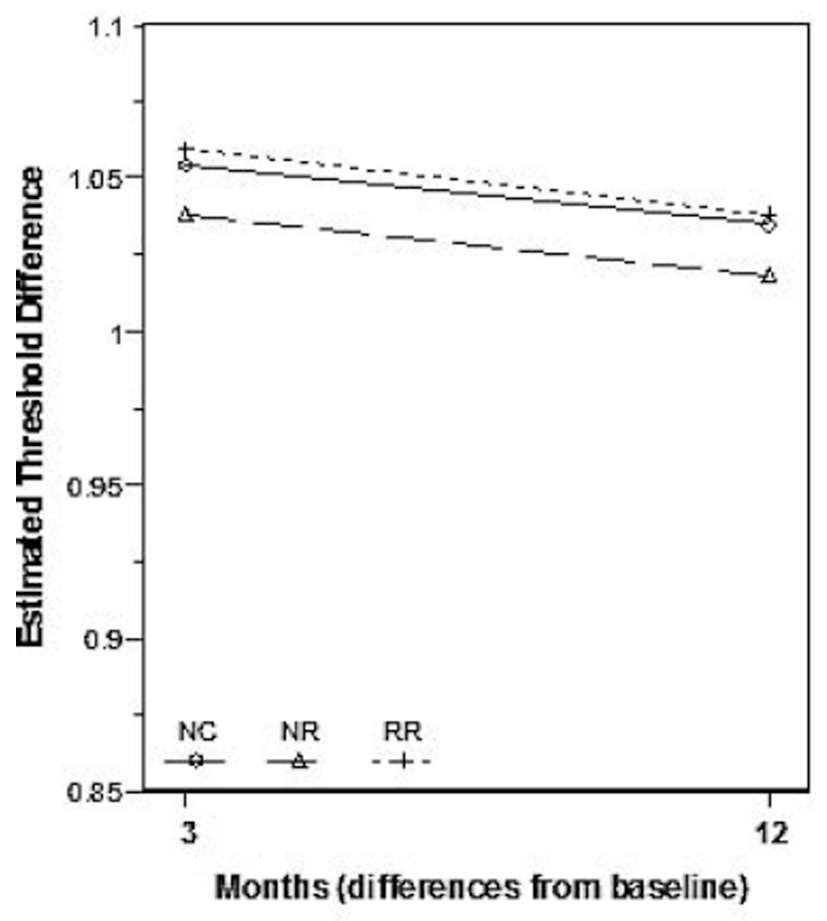

Male

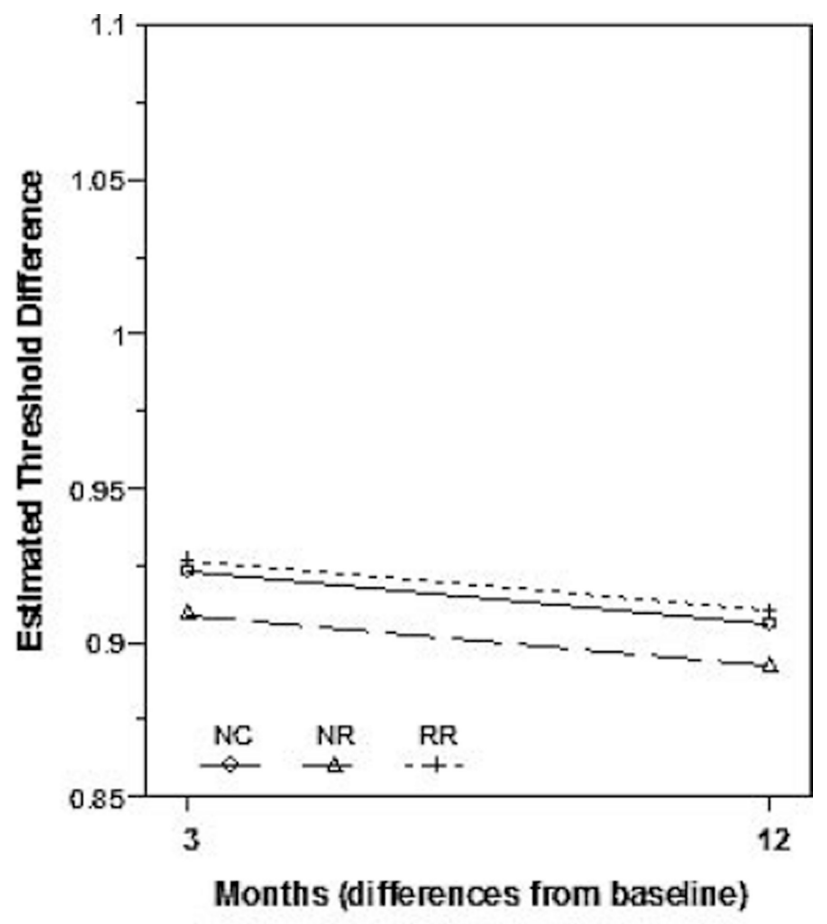

Female

FIGURE 1.

Estimated two-point perception impairment by group. Shown are the ratios of the geometric mean thresholds from each of the follow-up visits to the baseline visit, separately for male and female patients at the mean age at enrollment (15.84 years). 

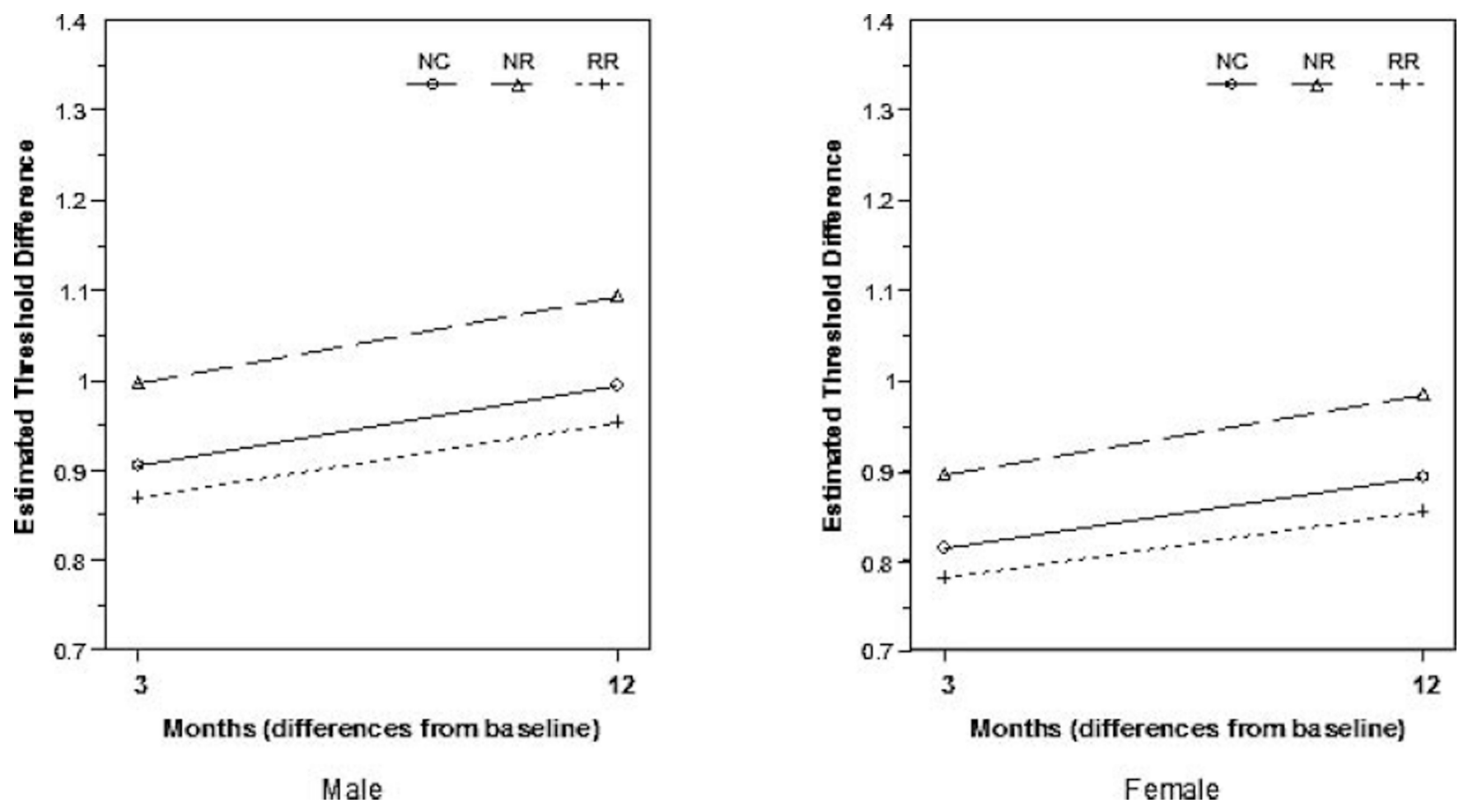

FIGURE 2.

Estimated warmth detection threshold impairment by group. Shown are the ratios of the geometric mean thresholds from each of the follow-up visits to the baseline visit, separately for male and female patients at the mean age at enrollment ( 15.84 years). 

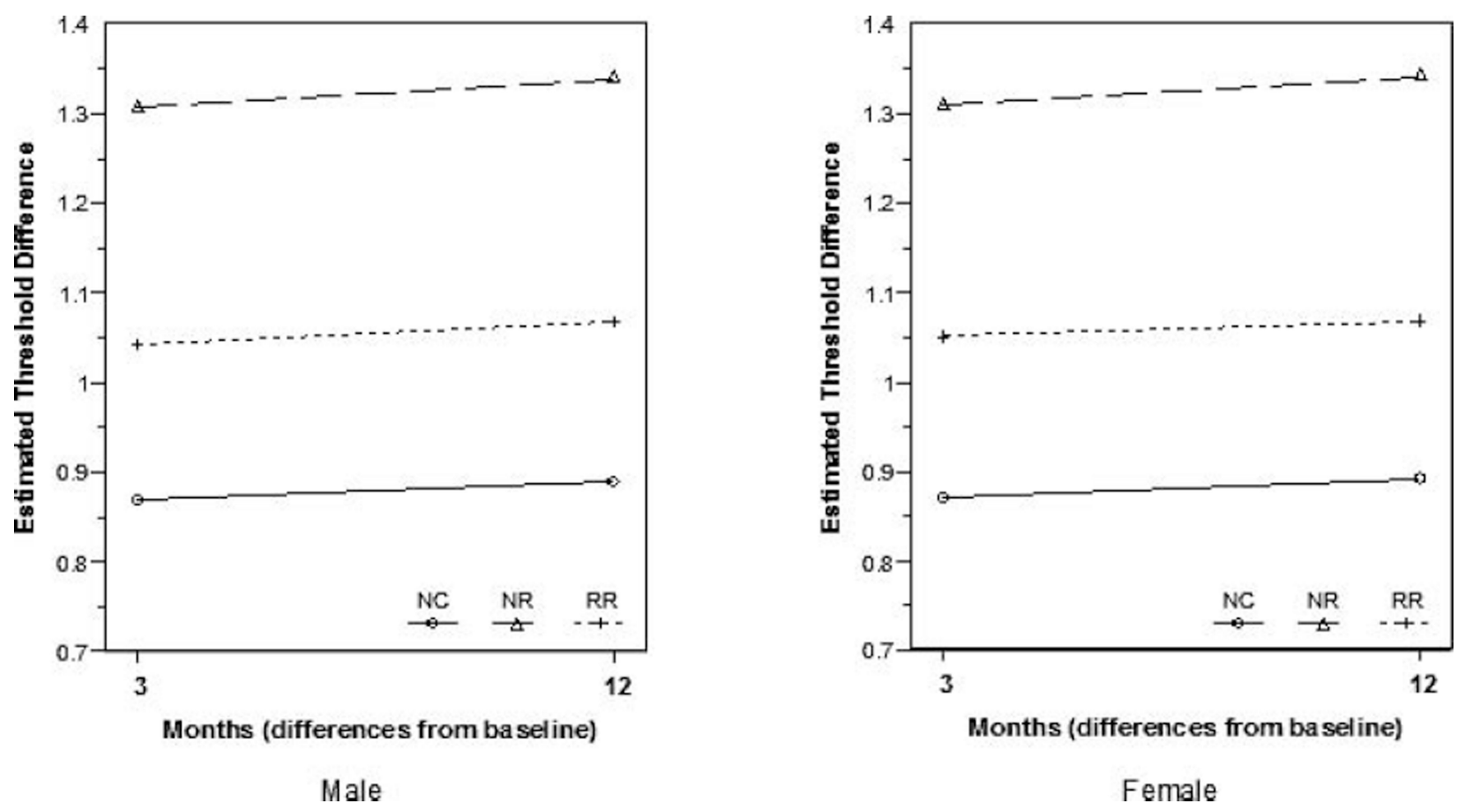

FIGURE 3.

Estimated cool detection threshold impairment by group. Shown are the ratios of the geometric mean thresholds from each of the follow-up visits to the baseline visit, separately for male and female patients at the mean age at enrollment ( 15.84 years). 


\section{TABLE 1}

Demographic Characteristics of Participants

\begin{tabular}{lrrrr}
\hline Group & N & \% Male & $\begin{array}{c}\text { Age, y } \\
\text { Mean }\end{array}$ & SD \\
\hline Non-cleft (control) & 22 & 55 & 15.7 & 2.7 \\
Revision & 20 & 65 & 16.1 & 2.5 \\
Without cleft palate & 4 & 25 & 16.6 & 3.6 \\
With cleft palate & 16 & 75 & 15.9 & 2.3 \\
Unilateral & 17 & 65 & 16.0 & 2.7 \\
Bilateral & 3 & 67 & 16.2 & 0.6 \\
Non-revision & 13 & 77 & 15.8 & 2.1 \\
Without cleft palate & 3 & 100 & 18.7 & 1.8 \\
With cleft palate & 10 & 70 & 15.0 & 1.1 \\
Unilateral & 12 & 75 & 15.7 & 2.1 \\
Bilateral & 1 & 100 & 17.1 & - \\
\hline
\end{tabular}


TABLE 2

Descriptive Statistics for the Log10 Difference Between 3 Months (12 Months) and Baseline for Two-Point Perception, Cool and Warmth Detection Thresholds by Group

\begin{tabular}{lllrr}
\hline Threshold & Group & $\begin{array}{r}\text { Baseline } \\
\text { Mean (SD) }\end{array}$ & $\begin{array}{r}\text { 3 Months- Baseline } \\
\text { Mean (SD) }\end{array}$ & $\begin{array}{r}\text { 12 Months- Baseline } \\
\text { Mean (SD) }\end{array}$ \\
\hline Two-point perception & Control & $0.520(0.110)$ & $-0.004(0.106)$ & $-0.012(0.126)$ \\
& Revision & $0.491(0.164)$ & $0.008(0.175)$ & $-0.007(0.151)$ \\
& Non-revision & $0.537(0.164)$ & $-0.003(0.119)$ & $0.001(0.125)$ \\
Warm & Control & $0.319(0.252)$ & $-0.047(0.251)$ & $-0.041(0.246)$ \\
& Revision & $0.436(0.300)$ & $-0.076(0.350)$ & $-0.036(0.324)$ \\
& Non-revision & $0.395(0.365)$ & $-0.043(0.171)$ & $0.061(0.294)$ \\
Cool & Control & $0.186(0.207)$ & $-0.058(0.150)$ & $-0.050(0.195)$ \\
& Revision & $0.213(0.285)$ & $-0.023(0.219)$ & $0.067(0.281)$ \\
& Non-revision & $0.146(0.312)$ & $0.166(0.228)$ & $0.078(0.178)$ \\
\hline
\end{tabular}




\section{TABLE 3}

$P$ Values Associated With the Main Effects for the Three Thresholds

\begin{tabular}{lccc}
\hline Effect & $\begin{array}{c}\text { Two-Point } \\
\text { Perception }\end{array}$ & $\begin{array}{c}\text { Warmth } \\
\text { Detection }\end{array}$ & $\begin{array}{c}\text { Cool } \\
\text { Detection }\end{array}$ \\
\hline Group & 0.98 & 0.81 & 0.04 \\
Age & 0.55 & 0.99 & 0.54 \\
Gender & 0.10 & 0.54 & 0.98 \\
Time & 0.64 & 0.19 & 0.72 \\
\hline
\end{tabular}

